\title{
Emergence of Methicillin Resistant S. epidermidis Isolates Causing Blood Stream Infections in Pediatric Patients
}

Aydın Aydınlı, Deniz Sertel Şelale, A. Demet Kaya

Okan University, Faculty of Medicine, Department of Medical Microbiology, Istanbul,Turkey

Background: Blood stream infection (BSI) is an important cause of morbidity and mortality in pediatric patients. Prompt and appropiate antimicrobial therapy can make the difference between cure and death or disability. Emergence of antimicrobial resistance, particularly methicillin resistance, in $S$. epidermidis is a serious problem. The aim of our study was to evaluate the causative agents of BSIs and antimicrobial susceptibility patterns of S.epidermidis strains, retrospectively.

Materials \& Methods: Blood samples were collected from neonatal intensive care unit and pediatric services of Okan University Hospital from JanuaryDecember 2017. Samples were evaluated with BacT / ALERT (Biomérieux, France) blood culture system. Catalase and coagulase tests (Plasmatec, England) were initially performed for the isolates associated with bacteremia. Vitek 2 Compact (Biomérieux, France) system was used to identifiy coagulase negative staphylococci and to determine their susceptibility to antimicrobials.
Results: Three hundered and ninty eight blood samples were sent for culture. Out of 398 samples, microbial growth was detected in 47 (11.8\%). Forty two (89.4\%) samples were considered to be associated with bacteremia, and 5 (10.6\%) as contamination.

Of the 42 isolates, 23 (54.8\%) were obtained from the pediatric service and $19(45.2 \%)$ from the neonatal intensive care unit. The most frequently isolated microorganism was S.epidermidis (27/42, 64.3\%),followed by Candida spp. (5/42, 11.9\%).

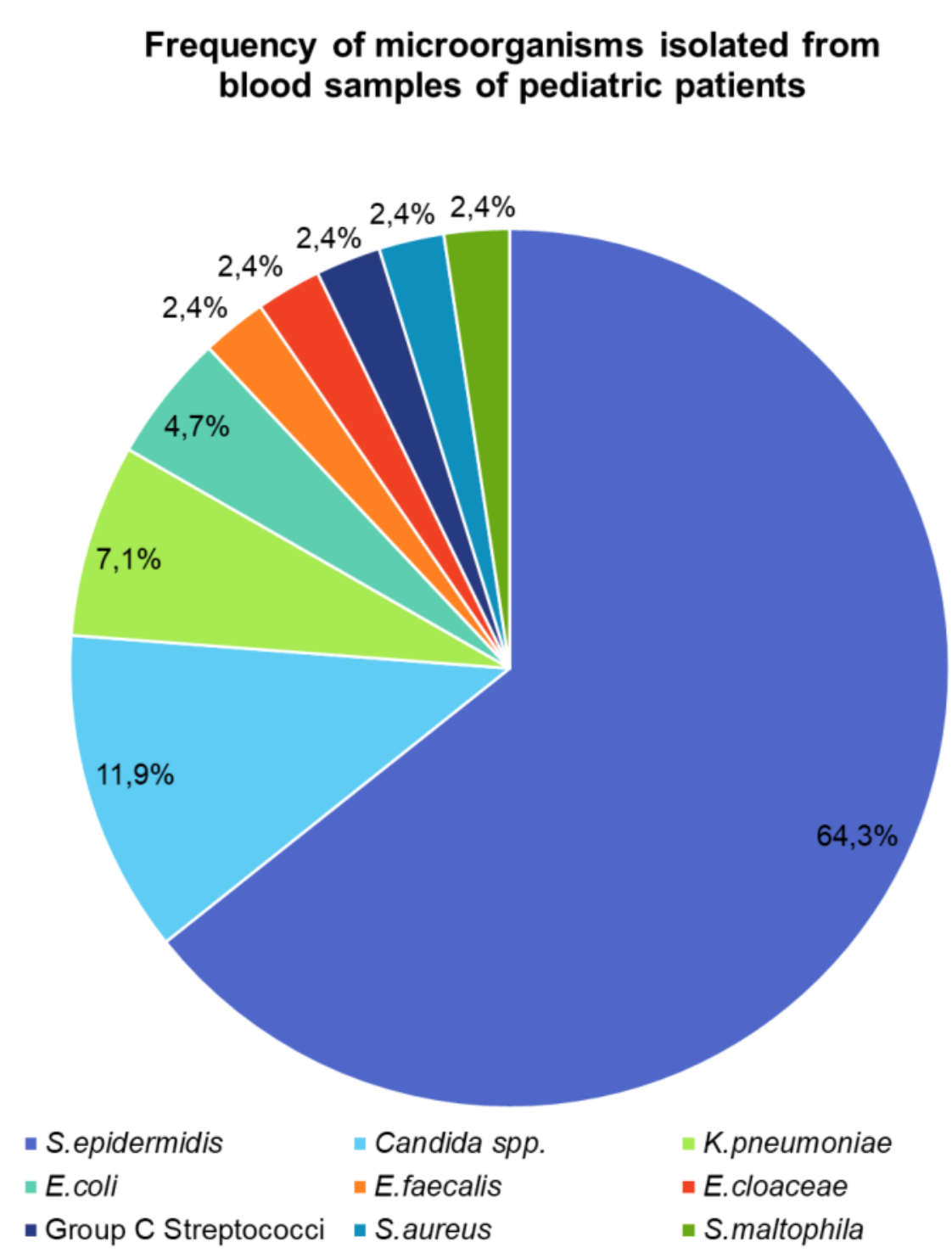

Other bacteria isolated from cultures were; K.pneumoniae (3/42, 7.1\%), E.coli (2/42, 4.7\%), E.faecalis (1/42, 2.4\%), E.cloacae (1/42, 2.4\%), Group C Streptococci $(1 / 42,2.4 \%)$, S. aureus $(1 / 42,2.4 \%)$ and S.maltophila (1/42, $2.4 \%)$.

All 27 S.epidermidis isolates (100\%) were resistant to methicillin (MET) and identified as MRSE and 10 of them $(37 \%)$ were obtained from samples sent from neonatal intensive care unit.

Resistance rates

S.epidermidis strains to other antibiotics were as follows; erythromycin (ERY) $85.2 \%$, clindamycin (CLI) $85.2 \%$, inducible clindamycin (InCLI) 92.6\%, phosphomicine (FOF) 92.6\%, trimethoprim/sulfomethoxazole (SXT) $85.2 \%$ and fusidic acid (FD) $96.3 \%$.

Conclusions: Rapid initiation of antimicrobial therapy is of high importance in bloodstream infections. Knowing the frequencies and susceptibility patterns of common microbial pathogens is crucial for selecting appropiate empiric therapy or prophylaxis. In our study high prevelance of methicillin and multi-drug resistance in S.epidermidis strains isolated from pediatric patients, emphasized the importance of continuous screening for antibiotic resistance in pediatric care units.

Keywords: Blood stream infection, $S$. epidermidis, antimicrobial resistance

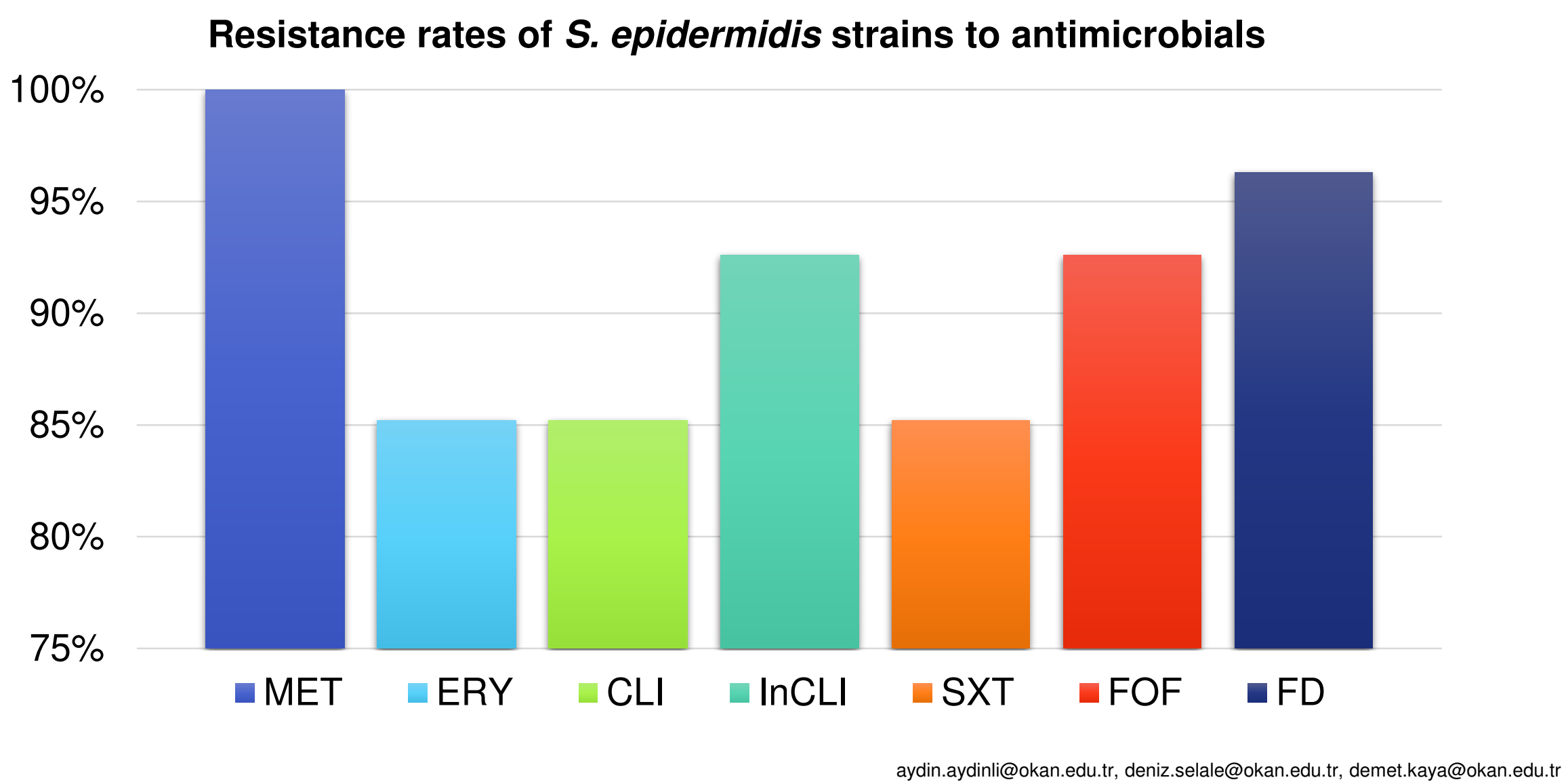

